\section{The challenges faced by service providers on satellite mental health-care facilities in Yangon, Myanmar: a qualitative study}

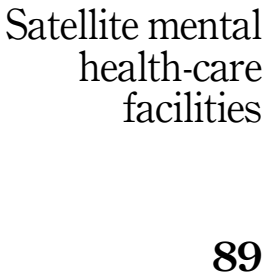

Kyaw Lin

Health Science, STI Myanmar University, Yangon, Myanmar

Sun Lin

Mental Health Unit, Nay Pyi Taw General Hospital, Nay Pyi Taw, Myanmar, and Than Tun Sein

University of Public Health, Yangon, Myanmar
Received 6 February 2020

Revised 18 May 2020

29 May 2020

10 June 2020

Accepted 15 June 2020

\begin{abstract}
Purpose - Myanmar has an insufficient number of mental health workers with few institutional facilities resulting in a significant treatment gap. Although few mental health services are integrated into primary health care (PHC), the challenges are unknown. This study aimed to assess the challenges perceived by providers in the service delivery of satellite mental health care (SMHC) in two sample townships in Yangon.

Design/methodology/approach - The research was based on a case study design by applying a qualitative approach using in-depth interviews (IDIs). In the three types of service providers, a total of six staff participated as interviewees. These consisted of two team leaders, two clinical specialists providing consultations to clients and two mental health nurses.

Findings - Providers perceived the following as major challenges in the provision of services: unstable financial resources and management, insufficient human resources and capacity of service providers, restricted outpatient services, the lack of a functional referral system, overcrowding, inadequate individual consultation time, long-waiting hours, finite opening days and hours and poor setting of infrastructure, resulting in lack of privacy.

Research limitations/implications - In the absence of similar studies in Myanmar, findings could not be placed in the context of the national literature for comparison. Further, the study involved a limited number of respondents, which may have affected the findings.

Originality/value - Although the challenges revealed were not uncommon in mental health services in developing countries, this study focused on a specific model of mental health care integrated into general
\end{abstract}

(C) Kyaw Lin, Sun Lin and Than Tun Sein. Published in Journal of Health Research. Published by Emerald Publishing Limited. This article is published under the Creative Commons Attribution (CC BY 4.0) licence. Anyone may reproduce, distribute, translate and create derivative works of this article (for both commercial and non-commercial purposes), subject to full attribution to the original publication and authors. The full terms of this licence may be seen at http://creativecommons.org/licences/by/4.0/ legalcode

The authors would like to thank the Medical Superintendent of Yangon Mental Health Hospital for granting permission to conduct interviews with the service provider teams of Satellite Mental Health Care Centers. With special gratitude to Professor Tin Oo, Head of Mental Health Department of the University of Medicine-1, Yangon and his staff for arranging interviews successfully.

Availability of data and materials: The full transcripts of all six IDI and six written informed consents of the participants in Myanmar language are available on request.

Funding: The authors declare that no funding was received from any individual or organization for this study. 
JHR

36,1

90

healthcare settings in Myanmar. The findings offer a benchmark on efforts to develop decentralized mental health services in Myanmar and provide input for future in-depth studies.

Keywords Integrated mental health services, Mental health care, Myanmar

Paper type Research paper

\section{Introduction}

Mental health continues to be one of the most neglected public health issues despite the increasing prevalence of mental health disorders worldwide. Mental health services can be classified into three main types: community-based services, services integrated into primary care and specialized institutional or hospital services [1] (Figure 1).

Individual countries may face different challenges depending on the types of mental health services provided. In many developing countries, restricted human resources [2], inaccessibility to mental health professionals [3] and inappropriate health-care settings [4] are found to be common challenges. Furthermore, mental health hospitals are notorious for their stigmatization [1] and violation of basic human rights [1,5]. Besides stigma, other important challenges include the following: low ranking as a health system priority, inadequate capacity in management, insufficient training of general practitioners (GPs), the absence of orientation to integrated care, a high turnover of staff, inefficient intersector coordination, poor infrastructure and insufficient budget allocation [4].

In Myanmar today, provision for mental health remains under the colonial era Lunacy Act 1912. The Ministry of Health and Sports (MoHS) is the main government body responsible for providing mental health care in the country. It relies on two mental health hospitals in Yangon and Mandalay, 15 state and regional level specialist hospitals and some district-level general hospitals to provide mental health services. It has only 200 mental health specialists serving a population of 54 million. Despite a mental health policy integrated into the National Health Plan, the effectiveness of mental health services is questionable because of overcrowded mental health hospitals and the lack of fully functional alternative mental health services, the absence of prevention and rehabilitation programs and the lack of community-based mental health services.

According to the Myanmar Mental Health Society, the treatment gap among people with mental illness in Yangon was over 85\% [6]. Stigma, lack of awareness, a neglected mental health service system and lack of access are shaping the treatment gap. Similar to other developing countries, an insufficient number of mental health staff and limited institutional facilities also apply in Myanmar. The MoHS collaborated with the WHO in the provision of training for more than 500 medical doctors and front-line health staff to be able to provide community-based mental health services [7]. However, in the absence of follow-up and evaluation, it remains difficult to judge its effectiveness.

Figure 1.

Different types of mental health services in a country, WHO [1]

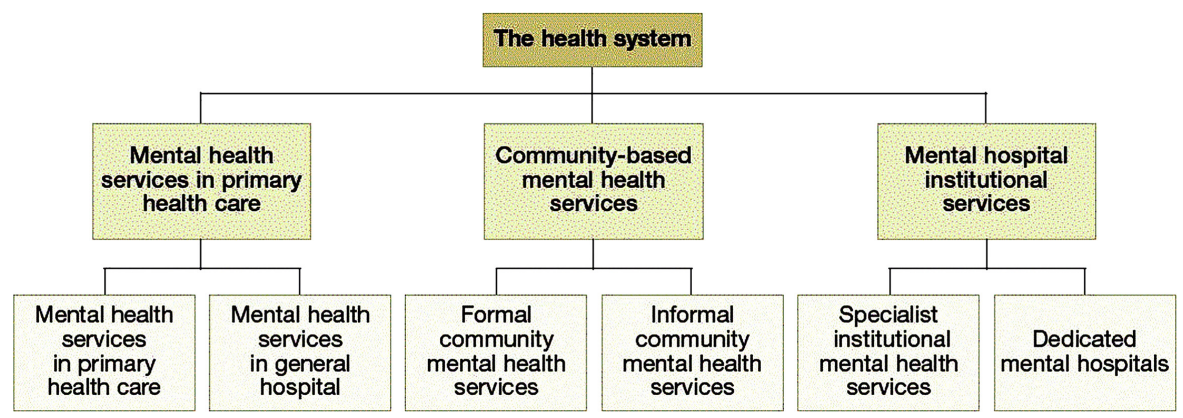


To fill the treatment gap in the Yangon Region, the satellite mental health care (SMHC) model was introduced in a few townships. Mental health professionals are implementing this mental health service model in some township hospitals in the satellite areas of the Yangon Region. Assisted by small financial contributions and donations from interested individuals and the community, a few mental health specialists from Yangon Mental Health Hospital (YMHH) launched the SMHC model on a voluntary basis in 2013. The YMHH supported service delivery with a certain amount of medications. Initially, it provided outpatient services in township general hospitals, trained GPs, and promoted mental health education in the community. However, because of different impediments, the services were gradually limited to medical treatment for mentally ill clients only. To strengthen service delivery, it was essential to identify the challenges of SMHC and identify pathways to overcome its barriers. This study focused on the identification of challenges in the provision of services from the providers' perspective in two SMHC sites in Yangon.

\section{Methodology}

A case study design was adopted by applying a qualitative descriptive approach to identify challenges [8] as perceived by SMHC service providers in Yangon, Myanmar.

\section{SMHC sites' selection}

The SMHC sites selected were Kyauktan and Kawhum townships in Yangon region because these two sites were among the longest established SMHC sites in Myanmar. The Kyauktan and Kawhmu townships are situated in 22 and 38 miles southeast of Yangon [9].

\section{Sampling}

This study used purposive sampling, which allowed for the inclusion of different types and levels of respondents [8]. The sample consisted of six respondents, including two team leaders responsible for planning and managing SMHC, two mental health specialists and two mental health nurses directly involved in SMHC service delivery in the Kyauktan and Kawhum townships. The sample size was based on the number of respondents expected to yield meaningful extrapolation about the studied population [8].

The distribution of respondents by location, level and type is summarized in Table 1 .

Among $\mathrm{MH}$ specialists and $\mathrm{MH}$ nurses, those who had provided the longest duration of service in each SMHC were selected, as it was deemed that they had the most complete organizational memory.

\section{Research tool}

To extract the experiences, perceptions and understanding or reasons of an issue from study participants [10], a semi-structured questionnaire was used to facilitate in-depth interviews (IDIs). The questionnaire covered major components of service and allowed many new useful areas to be discussed between the interviewer and respondent [11].

Major questionnaire components were derived from a description of services delivered. For example, types and characteristics of services, including waiting time, consultation time,

\begin{tabular}{lccc}
\hline Types of SMHC service provider & Kyauktan & Kawhmu & Total \\
\hline Team leader & 1 & 1 & 2 \\
MH specialist & 1 & 1 & 2 \\
MH nurse & 1 & 1 & 2 \\
Total & 3 & 3 & 6
\end{tabular}

Table 1.

Types and numbers of

SMHC service providers 
JHR

36,1

92

service hours and service charges, access to services such as economic, cultural, geographic and functional factors and the capacity of service providers, especially qualifications and deployment of health professionals, are responsible for service delivery. The translation of the IDI guidelines from English to the Myanmar language was crosschecked by two senior coresearchers.

\section{Data collection}

Interviewees received information on the research study, the purpose of the study, the nature of the interview and gave informed consent voluntarily before the IDI began. The interviewer conducted face-to-face interviews in a private place at the YMHH. With permission from interviewees, the interviews were audio recorded. The average duration of each IDI was approximately one hour. All interviews were conducted in March 2019.

\section{Data management and analysis}

Transcribed qualitative interview data in the Myanmar language served as a latent content analysis [12]. Content analysis was facilitated by ATLAS. ti8 software, using a mix of predefined codes aligned with IDI questionnaire components. During the analysis, codes were modified based on evolving codes (hybrid coding). Individual findings were triangulated across respondents and participating SMHC. Verbatim quotes were translated into the English language.

\section{Ethical considerations}

Ethical approval was obtained from two institutes, the Institutional Review Board of Defense Services Medical Research Centre, Directorate of Medical Services, Ministry of Defense, Nay Pyi Taw, Myanmar (IRB/2019/6) and the Institute for Health Research Ethics Committee, University of Bedfordshire (IHREC918) in February 2019.

\section{Results}

\section{Respondent profiles}

In brief, four females and two males aged between 29 and 45 years participated in the study. Professional experience of participants working in SMHC ranged from one to five years.

\section{Current status of SMHC service delivery in two townships}

During the initial years, the total number of outpatient clients was in the range of two digits only, which was later increased to three digits. At the time of the study, the number of active registered outpatient clients was 300 to 400 in the Kyauktan center and 150 to 200 in the Kawhmu center. The centers served clients suffering from a variety of mental health disorders ranging from alcohol and substance abuse, anxiety, mood and psychotic disorders. The number of male clients was slightly higher than female clients. The majority of male clients presented with alcohol and substancerelated illnesses, while female clients commonly presented with anxiety disorders. The majority of clients ranged between 30 and 40 years of age. Based on the respective local general hospitals, the mental health services were delivered on a single day in every second or third week of a month.

Financial support and management. Because the SMHC relied on contributions from individuals and other small charities, the financial resources were insecure. No specific funding or health insurance system was in place.

Now, it looks like we are depending only on individual donations, without any big organizations except "XYZ" Social Support Group. But they contribute only occasionally. Respondent-2 
"If we have a health insurance system, it may be better because that is essential for a good health service. Respondent-1

To overcome financial difficulties, collecting cash from clients was not in line with the MoHS policy.

If we cannot get the government funding in between, I would like to collect 1000 or 1500 Kyats (0.6 1 USD) from each client who comes for service, like a donation to the service. However, if we provide this service and collect money from clients against government regulations, it may become complicated. Respondent-2

Although funding from donors or nongovernmental organizations (NGOs) was an alternative option, the interest of funders and NGOs on mental health was low.

They (NGOs) have their own interests. They are more enthusiastic about communicable diseases (CD). Mental health is listed under non-CD, and so they are less aware of mental health. Respondent-1

The practical long-term solution was a commitment from the government to allow more official funding for mental health.

The government has to keep in mind that community-based mental health programs should exist because it is necessary. Respondent-1

Human resources. A shortage of staff, especially mental health nurses, was reported. Moreover, a lack of other types of mental health professionals was also noticed. Insufficient nurses and other professionals could be handled by the MoHS.

We need more staff, senior and junior nurses because we have to provide medication. If there is no medication, we may not be needed. Respondent- 5

But the main issue is nurses; there is no big number of nurses. Even when a specialist is available, there is no nurse to assist. Here, we have a shortage of nurses, both seniors and juniors. Respondent-3

Because the existing service program was not fully endorsed by the MoHS, it was difficult to appoint more and different mental health staff to strengthen the SMHC or any potential expansion of service. Retraining GPs was considered an alternative option.

We have not talked to them (GPs) yet. Would they be able to prescribe and provide the required medication, if we refer patients to them? The number of patients with mental health issues is lower than those of general patients. They need to keep some medication for them anyway. The main requirement is its acceptability. To train them, we need more funding and monitoring and supervising too. Respondent-2

Another alternative option raised was to collaborate with NGOs in sharing skills and services.

Another option is, in case of any interested NGO, we can provide medication and they provide other services (psychosocial and counseling). Such cooperation may be better. Respondent-1

Capacity of service providers. Some postgraduate students of mental health specialty required more skills and training in taking care of their clients. Due to limited time and skills, it was insufficient in capacity.

Technically, they should provide psychotherapy, relaxation or breathing exercise to clients with sleep or anxiety problems. It is a lot better to take time and explain to the clients how to take care of themselves. Respondent-2

They all have a certain degree of knowledge and skill. But in some complicated cases, they need to handle it more wisely. It depends on the individual. While some providers have an interest and give 
JHR

36,1

care more precisely, others are less interested and unable to adjust the medication and make poor assessments of the clients. Respondent-1

Deploying only qualified postgraduate specialists was seen as a solution to improve the quality of care.

Previously, we appointed postgraduate students. Because they had some shortcomings in adjusting the prescribing medication, we now deploy only those who have a postgraduate degree. Respondent-2

Types of services provided. At the time, the service provided limited but free medical treatment for outpatients without psychosocial support, counseling for clients or caregivers. No prevention or promotion activity was included because of several constraints.

We provide certain essential types of medications free of charge. The main criteria set for such medications are good efficacy with a reasonable price range. Respondent-3

Good case management in the community, especially for those discharged clients, GP-led mental health services and social and occupational support was also regarded as essential services to expand.

It should have linkage. If a patient is discharged from the hospital, the community team should contact that person. Now, if a patient is discharged, we do not know what happens to that person. Ideally, the patient is cared for and watched by the community team. Later, contact us again (if needed). If such a channel develops, it is good. What kinds of resources are available in that person's community, for example, any occupational opportunity? Then, the team contacts the source of the job and the patient can be employed; social support should be continued. In my opinion, it can reduce the stigma on them too. Respondent-1

Service characteristics. Only minimal services could be provided and could not meet the demand within the community. Overcrowding, restricted consultation times for individual clients and poor service infrastructure settings of service delivery were reported by interviewees.

We provide only minimal care but not of high quality. As you know, we are providing services in the local community: if you take too much time on a single client, others will have to wait unnecessarily long. Moreover, it lacks privacy too: if you interview someone, others can hear about it. Respondent-2

Our care is not enough. Why? Because of time and manpower limitations, we cannot provide what we ought to. For instance, with a new client, we need to talk to them for at least 20 minutes. We cannot even provide it. With a total of 400 clients, we sometimes listen to them; sometimes we have to interrupt and terminate the conversation prematurely, so it (the contact time) is only less than 5 minutes per client. Respondent-3

The lack of a functional mental health referral system made undue overcrowding of service centers.

If we have a "cascade" type of referral system, the workload for the clients may reduce and the quality (of care) can improve. Respondent-2

When completing a mental state examination, there is not enough space. There are only two tables for four medical officers to sit around. A lot of clients surround this space, and there is no private space. Respondent-3

The SMHC faced an inadequate setting of service provision, which depended on the host hospitals.

The present service delivery room provided for us is practically a meeting room and is the widest space in the township general hospital. Sometimes, it is noisy because of the large crowd of clients; we have to shout. What the medical superintendent of the hospital allows us is his best. Respondent-3 
Service eligibility and accessibility. Any type of mental health client was eligible to make use of the service. The centers were situated in the respective township hospitals, with service offered every two or three weeks and no restriction on the catchment area was determined.

They (general hospitals) refer clients to us and we provide treatment for them. They visit us freely, and no documents or referral letters are needed. Some are self-referred just for sleep problems. We do not fix the (eligibility of) catchment areas. Respondent-3

Respondents believed that there was no problem in terms of accessibility and availability of mental health services.

Because we provide a free service to all clients, I think there is no major challenge in accessibility and availability of service from a provider perspective. Respondent- 4

On service delivery day, the available consultation hours were rather limited. This was explained by the required time for the SMHC team to travel from Yangon to the respective satellite townships. As a result, service delivery could not start earlier in the morning.

The main issue is time. From Yangon, we travel by road and when we get there, it is almost 10:30 a.m. After making everything ready for the service, we start providing treatment until 3 p.m. Respondent-3

Communication. All service providers, clients and caregivers spoke Myanmar language. However, communication between service providers and clients/caregivers became at times impersonal due to workload-related stress.

Sometimes, we shout at them (clients/caregivers) when they do not come for their turn. Then, they eagerly try to get their (clinic) record book back (from us) without properly checking it. Good communication with clients is almost absent, as we have no time. Within a very few hours, we have to rush everything. Often, they are also upset for queuing and talk back to us. Respondent- 6

\section{Discussion}

Although controversial, the recent influence of western mental health, its principles, classifications and treatments dominates the global mental health movement to raise awareness and promotes treatment to fulfill the mental health needs in developing countries and vulnerable groups in developed countries [13].

Acknowledging the variances across societies, the mental health treatment gap is found to be over $90 \%$ in developing countries [14] where the numbers and types of mental health services are insufficient and often of poor quality.

Narrowing the mental health treatment gap in developing countries cannot be achieved by upgrading mental health facilities but developing existing mental health systems through integration of mental health care into primary health care (PHC) settings [1]. The WHO recommended this strategy as it would act as a crucial link between specialized mental health hospitals and community-based services. In line with the WHO [1] recommendations, the SMHC model under study offers limited mental health services in secondary level general hospitals or primary health-care settings.

As a sound financial management policy was absent, financial insecurity was reported by service providers as a major challenge. Although the MoHS was the chief funder for public mental health services in Myanmar, the SMHC faces a lack of formal and secure financial resources. Financial hardship [15] is recognized as a crucial challenge in mental health services. However, where resources are limited, the way resources are allocated and used is the key factor for enhancing efficiency and effectiveness [16]. To overcome financial hardship, the financial resources for mental health ought to rely not only on charitable sources but also on public funding or individual collectives as a whole [17]. The SMHC in this 
JHR

36,1 study had to rely on NGOs and charitable donations. To overcome financial insecurity and enhance sustainability, government budget allocation for SMHC is important.

Another challenge reported was insufficient manpower. The SMHC team consisted of psychiatrists and $\mathrm{MH}$ nurses only. Besides the inadequacy of $\mathrm{MH}$ nurses, other types of mental health workers, such as psychologists, counselors, social workers and occupational therapists, are lacking. This limitation in human resources impacts the quality of care. The MoHS in Myanmar is the sole responsible body for the development of a mental health workforce. The production of a sufficient workforce, size is a long-term solution, for the shortterm "task-shifting" and capacity building among nonprofessionals in community-based mental health services may provide a viable strategy [2]. However, to do so, barriers posed by psychiatrists and mental health leaders, who have a very limited understanding of public health, need to be overcome [18]. To change attitudes among mental health specialists, topics on public mental health should become part of the medical and specialist curricula.

Because of the multitude of challenges, including financing, human resources, infrastructure, organizational issues and the absence of caregivers' support, SMHC can only provide very limited services.

Owing to the various limitations of the mental health system in Myanmar, the provision of $\mathrm{SMHC}$ is insufficient to address these shortcomings. Services by multidisciplinary teams need to be developed at tertiary, secondary and primary care levels for outpatients and inpatients and complemented by community-based services. Specific supportive services for caregivers, such as mental health literacy and assistance in coping with stress, should also be developed.

The combination of SMHC challenges inevitably affects the quality of care. Budget, infrastructure, human resources and organizational limitations result in insufficient consultation time, stressed-out service providers, sole clinical treatment and lack of attention for caregivers. As argued in the literature, the quality of care is a crucial requirement in the provision of mental health services and depends upon multiple factors. For example, in rural India, because of insufficient funding, human resources and facilities for service settings, the quality of mental health care was found to deteriorate [18]. It is advised to base quality of care not only on currently approved care for mental health clients, but similar standards must be used to address the needs of the affected community to relieve their burden and to improve the quality of communal life [19]. Indeed, the outcome of a client's mental health condition is closely related to the coverage and quality of services provided [1].

To relieve the financial burden of patients and families, many countries pursue a decrease in out-of-pocket expenses (OOPE) [20]. Providing free medication and service that would ameliorate OOPE by reducing expenditure is favored by some authors [21]. Nonetheless, the free supply of medication is a huge financial issue for service providers, especially when financial resources are uncertain and limited. Moreover, it may undermine the sustainability of the service provided. In recognition of these realities, the WHO [20] suggested the practice of combining prescriptions with the availability of medication through cost-effective procurement plans, mass buying, increased focus on generic drugs and no free of charge medication but appropriate cost-sharing by the clients.

\section{Limitations}

The design of our study has its own limitations. In the absence of other methods, triangulation was limited to IDI respondents and location. The sample was rather small, involving only six staff out of 10 to 14 and no inclusion of clients and/or caregivers, which may have affected the findings. However, this study offers a benchmark on efforts to integrate mental health services in PHC settings in Myanmar through SMHC. 
Conclusion and recommendations

The identified challenges of two SMHC in the Yangon Region, Myanmar were as follows: limited and unsecured financial resources, insufficient human resources, limitations in terms of infrastructure and organization and a dysfunctional referral system. These limitations affected the quality of services offered in terms of sizable patient load for limited consultation time. Providers become stressed when coping with the workload, which then adversely affects provider-patient/caregiver communication. Although these challenges, their underlying reasons and their effects on the quality of mental health services are commonly acknowledged across developing countries, this study evidences efforts within Myanmar to develop decentralized mental health services and the struggles in doing so.

Future in-depth studies are needed by involving decision-makers at the health system level, clients and caregivers, community-based groups, affected communities and policy actors by employing multiple methods. This is to enhance a wider comprehension of mental health service needs, challenges and pathways in addressing these issues systematically.

Conflict of interest: There is no conflict of interest.

\section{References}

1. World Health Organization [WHO]. Organization of services for mental health. Geneva: WHO; 2003.

2. Kakuma R, Minas H, van Ginneken N, Dal Poz MR, Desiraju K, Morris JE. Human resources for mental health care: current situation and strategies for action. Lancet. 2011 Nov; 378(9803): 1654-63. doi: 10.1016/S0140-6736(11)61093-3.

3. Patel V, Belkin GS, Chockalingam A, Cooper J, Saxena S, Unutzer J. Grand challenges: integrating mental health services into priority health care platforms. Plos Medicine. 2013 May; 10(5): e1001448. doi: 10.1371/journal.pmed.1001448.

4. Marais DL, Petersen I. Health system governance to support integrated mental health care in South Africa: challenges and opportunities. Int J Ment Health Syst. 2015 Mar; 9: 14. doi: 10.1186/ s13033-015-0004-z.

5. Cohen A, Minas H. Global mental health and psychiatric institutions in the 21st century. Epidemiol Psychiatr Sci. 2017 Feb; 26(1): 4-9. doi: 10.1017/S2045796016000652.

6. Downing J. Mending troubled minds. [updated 2016 Oct 21; cited 2017 May 16]. Available from: http://frontiermyanmar.net/en/mending-troubled-minds.

7. The ASEAN Secretariat. ASEAN mental health systems. Jakarta: The ASEAN Secretariat; 2016.

8. Marshall MN. Sampling for qualitative research. Fam Pract. 1996 Dec; 13(6): 522-5. doi: 10.1093/ fampra/13.6.522.

9. The General Administrative Department. The statistics of the Yangon region 2017. Yangon: The General Administrative Department; 2017.

10. Milena ZR, Dainora G, Alin S. Qualitative research methods: a comparison between focus-group and in-depth interview. Annals of Faculty of Economics. 2008; 4: 1279-83.

11. Gill P, Stewart K, Treasure E, Chadwick B. Methods of data collection in qualitative research: interviews and focus groups. Br Dent J. 2008 Mar; 204(6): 291-5. doi: 10.1038/bdj.2008.192.

12. Leech NL, Onwuegbuzie AJ. Qualitative data analysis: a compendium of techniques and a framework for selection for school psychology research and beyond. School Psychol Quart. 2008 Dec; 23(4): 587-604. doi: 10.1037/1045-3830.23.4.587.

13. Cooper S. Global mental health and its critics: moving beyond the impasse. Crit Public Health. 2016; 26(4): 355-8. doi: 10.1080/09581596.2016.1161730.

14. Patel V, Maj M, Flisher AJ, De Silva MJ, Koschorke M, Prince M. Reducing the treatment gap for mental disorders: a WPA survey. World Psychiatr. 2010 Oct; 9(3): 169-76. doi: 10.1002/j.2051-5545. 2010.tb00305.x.
Satellite mental health-care facilities

\section{(}


15. Thornicroft G, Tansella M, Law A. Steps, challenges and lessons in developing community mental health care. World Psychiatr. 2008; 7(2): 87-92. doi: 10.1002/j.2051-5545.2008.tb00161.x.

16. Knapp M, Funk M, Curran C, Prince M, Grigg M, McDaid D. Economic barriers to better mental health practice and policy. Health Policy Plan. 2006 May; 21(3): 157-70. doi: 10.1093/heapol/czl003.

17. World Health Organization [WHO]. Mental health financing. Geneva: WHO; 2003.

98

18. Kumar A. Mental health services in rural India: challenges and prospects. Health. 2011; 3(12): 757-61. doi: 10.4236/health.2011.312126.

19. World Health Organization [WHO]. The mental health context. Geneva: WHO; 2003.

20. World Health Organization [WHO]. Improving access and use of psychotropic medicines. Geneva: WHO; 2003.

21. Ali SH, Agyapong VIO. Barriers to mental health service utilisation in Sudan - perspectives of carers and psychiatrists. BMC Health Serv Res. 2016 Jan; 16: 31. doi: 10.1186/s12913-016-1280-2.

\section{Corresponding author}

Kyaw Lin is can be contacted at: drkyawlin@gmail.com

For instructions on how to order reprints of this article, please visit our website: 